\title{
Towards a thermodynamics of active matter
}

\author{
S. C. Takatori and J. F. Brady" \\ Division of Chemistry and Chemical Engineering, California Institute of Technology, Pasadena, California 91125, USA
}

(Received 14 November 2014; published 11 March 2015)

\begin{abstract}
Self-propulsion allows living systems to display self-organization and unusual phase behavior. Unlike passive systems in thermal equilibrium, active matter systems are not constrained by conventional thermodynamic laws. A question arises, however, as to what extent, if any, can concepts from classical thermodynamics be applied to nonequilibrium systems like active matter. Here we use the new swim pressure perspective to develop a simple theory for predicting phase separation in active matter. Using purely mechanical arguments we generate a phase diagram with a spinodal and critical point, and define a nonequilibrium chemical potential to interpret the "binodal." We provide a generalization of thermodynamic concepts like the free energy and temperature for nonequilibrium active systems. Our theory agrees with existing simulation data both qualitatively and quantitatively and may provide a framework for understanding and predicting the behavior of nonequilibrium active systems.
\end{abstract}

DOI: 10.1103/PhysRevE.91.032117 PACS number(s): 05.70.-a, 47.63.Gd, 64.75.Xc, 05.65.+b

\section{INTRODUCTION}

Self-propulsion is a distinguishing feature of all "active matter" systems. By controlling and directing their own behavior self-propelled entities (usually, but not restricted to, living systems) can exhibit distinct phases with unusual dynamical properties [1]. These exotic behaviors are made possible because active matter is an inherently nonequilibrium system that is not bound by conventional thermodynamic constraints. A key challenge is to develop a framework for understanding the dynamic behavior and bulk properties of active matter.

While computer simulations have produced phase diagrams of active matter [2-6], many regions of phase space are difficult to explore because of the computational challenge of covering the parameter space. In this paper we develop a mechanical theory for predicting the phase behavior of active systems. We also offer suggestions on how conventional thermodynamic concepts, such as chemical potential, free energy, and temperature, can be extended to provide a "thermodynamics" of nonequilibrium active matter. At this point we are not certain whether conventional thermodynamic concepts comprise a valid and rigorous framework for studying nonequilibrium active systems. It remains uncertain to what extent, or even if, any of the concepts from classical thermodynamics are applicable to active matter. Our analysis suggests that active systems are entropically driven by a lower critical solution temperature (LCST) transition, where phase separation becomes possible with increasing temperature.

Here we consider a simple active matter system-a suspension of self-propelled spheres of radii $a$ that translate with an intrinsic swim velocity $\boldsymbol{U}_{0}$, tumble with a reorientation time $\tau_{R}$, and experience a hydrodynamic drag factor $\zeta$ from the surrounding continuous Newtonian fluid. The random tumbling results in a diffusive process for $t \gg \tau_{R}$ with $D^{\text {swim }}=U_{0}^{2} \tau_{R} / 6$ in three dimensions (3D). We do not include the effects of hydrodynamic interactions, and there is no polar order of the swimmers, precluding any large-scale collective

*jfbrady@caltech.edu motion (e.g., bioconvection). We seek to understand the phase behavior of a simple active system in which there is no large-scale coherent motion before moving on to study more complex collective behavior.

\section{MECHANICAL THEORY}

The active pressure exerted by a system of self-propelled particles can be written as $\Pi^{\text {act }}=\Pi^{\text {swim }}+\Pi^{P}$, the sum of the "swim pressure" $\Pi^{\text {swim }}$ and the interparticle (collisional) pressure $\Pi^{P}[7]$. It is permissible to add the separate contributions of the pressure in what appears to be a superposition; this is true in general for molecular, Brownian, and active systems.

The swim pressure was recently introduced as a fundamental aspect of active systems and as an aid to understand their self-assembly and phase behavior [7-9]. For a dilute system the "ideal-gas" swim pressure is $\Pi^{\text {swim }}=n \zeta D^{\text {swim }}=n \zeta U_{0}^{2} \tau_{R} / 6$ [7]. Physically, $\Pi^{\text {swim }}$ is the unique pressure exerted by self-propelled entities as they bump into the surrounding walls that confine them, analogous to the osmotic pressure of colloidal solutes. The swim pressure is an entropic quantity that arises purely from confinement, and can be computed from the first moment of the self-propulsive swim force (see Appendix A and B).

Dimensional analysis allows us to write the swim pressure as $\Pi^{\text {swim }}\left(k_{s} T_{s}, \phi, \mathrm{Pe}_{R}\right)=n k_{s} T_{s} \widehat{\Pi}^{\text {swim }}\left(\phi, \mathrm{Pe}_{R}\right)$, where $k_{s} T_{s} \equiv \zeta U_{0}^{2} \tau_{R} / 6$ defines the swimmers' "energy scale"force $\left(\zeta U_{0}\right) \times$ distance $\left(U_{0} \tau_{R}\right)$-and $\widehat{\Pi}^{\mathrm{swim}}\left(\phi, \mathrm{Pe}_{R}\right)$ is the nondimensional swim pressure that depends, in general, on the volume fraction $\phi=4 \pi a^{3} n / 3$ and importantly the nondimensional reorientation "Péclet number" $\mathrm{Pe}_{R}=U_{0} a / D^{\mathrm{swim}}=U_{0} a /\left(U_{0}^{2} \tau_{R}\right)=a /\left(U_{0} \tau_{R}\right)$, which is the ratio of the swimmer size $a$ to its run length $U_{0} \tau_{R}$ [10].

For large $\mathrm{Pe}_{R}$ the swimmers reorient rapidly and take small swim steps, behaving as Brownian walkers [7]. Thus $\widehat{\Pi}^{\text {swim }}\left(\phi, \mathrm{Pe}_{R}\right)=1$ for all $\phi \lesssim \phi_{0}$ where $\phi_{0}$ is the volume fraction at close packing. This system is analogous to passive Brownian particles, which exert the ideal-gas Brownian osmotic pressure $\Pi^{B}=n k_{B} T$ regardless of the concentration of particles. 
For small $\mathrm{Pe}_{R}$ the swimmers have run lengths large compared to their size and $\widehat{\Pi}^{\text {swim }}$ decreases with $\phi$ because the particles hinder each others' movement. In this limit experiments and computer simulations [6,11-15] have observed the self-assembly of active systems into dense and dilute phases resembling an equilibrium liquid-gas coexistence.

Extending the results of the nonlinear microrheology analysis [7] the swim pressure at small $\mathrm{Pe}_{R}$ in 3D takes the form $\widehat{\Pi}^{\text {swim }}=1-\phi-\phi^{2}$. The inclusion of a three-body term $\left(-\phi^{2}\right)$ is based upon an empirical fit which agrees with our swim pressure data for all $\mathrm{Pe}_{R} \leqslant 1$. Unlike Brownian systems where repulsive interactions (e.g., excluded volume) increase the pressure, for active matter interactions decrease the run length and therefore the swim pressure. The decrease in $\Pi^{\text {swim }}$ is the principle destabilizing term that facilitates a phase transition in active systems.

At finite concentrations, interparticle interactions between the swimmers give rise to an interparticle (or collisional) pressure $\Pi^{P}\left(k_{s} T_{s}, \phi, \mathrm{Pe}_{R}\right)=n k_{s} T_{s} \widehat{\Pi}^{P}\left(\phi, \mathrm{Pe}_{R}\right)$, where $\widehat{\Pi}^{P}\left(\phi, \mathrm{Pe}_{R}\right)$ is the nondimensional interparticle pressure. For repulsive interactions $\Pi^{P}$ increases monotonically with $\phi$ and helps stabilize the system. The phase behavior of active systems is determined by a competition between a destabilizing $\Pi^{\text {swim }}$ versus a stabilizing $\Pi^{P}$, a balance controlled by the parameter $\mathrm{Pe}_{R}$.

For large $\mathrm{Pe}_{R}$ the swimmers behave as Brownian particles and $\widehat{\Pi}^{P}\left(\phi, \mathrm{Pe}_{R}\right)=\widehat{\Pi}^{\mathrm{HS}}(\phi)$, where $\widehat{\Pi}^{\mathrm{HS}}(\phi)=4 \phi g(2 ; \phi)$ is the interparticle pressure of hard-sphere Brownian particles and $g(2 ; \phi)$ is the pair-distribution function at contact $[16,17]$. The detailed interactions between the particles are not important [16-18]; a hard-sphere molecular fluid's interparticle pressure has the same form-the same volume fraction dependence - as that of a Brownian system despite differences in the source of the collisions. A system of active swimmers also exhibits the same form of the interparticle pressure. Indeed, for large $\mathrm{Pe}_{R}$ the run length $U_{0} \tau_{R}$ sets the scale of the force moment and $\Pi^{P} \sim n^{2} \zeta U_{0} a^{3}\left(U_{0} \tau_{R}\right) \sim n k_{s} T_{s} \phi$, analogous to the passive hard-sphere Brownian collisional pressure $\sim n k_{B} T \phi$.

For small $\mathrm{Pe}_{R}, \Pi^{P} \sim n^{2} \zeta U_{0} a^{4} \sim n k_{s} T_{s} \mathrm{Pe}_{R} \phi$ since a swimmer is displaced by its size $a$ upon collision, not the run length $U_{0} \tau_{R}$. Extending the results of [7] the interparticle pressure for small $\mathrm{Pe}_{R}$ in $3 \mathrm{D}$ is thus $\widehat{\Pi}^{P}=3 \phi \mathrm{Pe}_{R} g(2 ; \phi)$.

For both small and large $\mathrm{Pe}_{R}$, the pair-distribution function at contact has the form [17] $g(2 ; \phi)=\left(1-\phi / \phi_{0}\right)^{-\beta}$, and $\phi_{0}$ and $\beta$ are parameters obtained from the interparticle pressure of hard-sphere molecular fluids and/or passive Brownian particles. Simulations verify that the parameters $\phi_{0}=0.65$ and $\beta=1$ agree independently with the collisional pressures for hard-sphere active swimmers, passive Brownian particles, and molecular fluids [17].

The active pressure is the sum of the swim and interparticle pressures [19], which for small $\mathrm{Pe}_{R}$ is

$$
\Pi^{\text {act }}=n k_{s} T_{s}\left[1-\phi-\phi^{2}+3 \phi \mathrm{Pe}_{R}\left(1-\phi / \phi_{0}\right)^{-1}\right],
$$

and which we can use to analyze phase separation in active matter. We focus on non-Brownian swimmers since the effect of translational Brownian diffusivity is small in phaseseparating systems. Figure 1 compares the phase diagram in the $\mathrm{Pe}_{R^{-}} \phi$ plane obtained from this model to the simulation data of other studies.
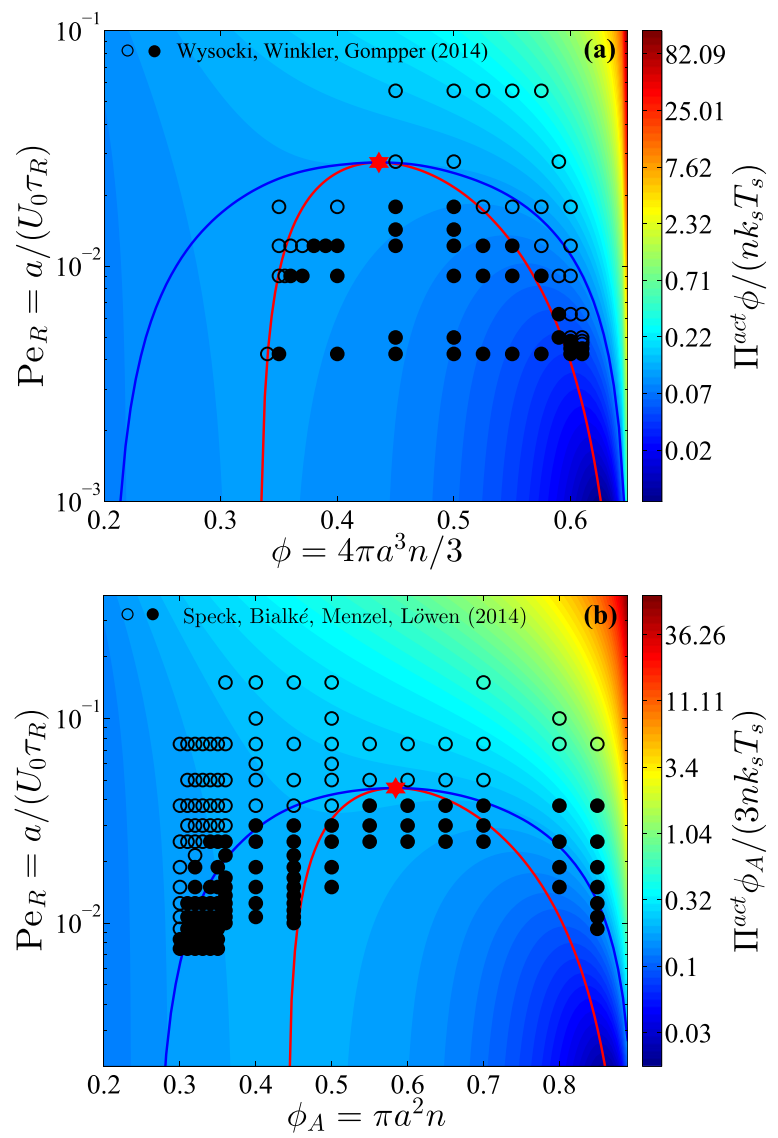

FIG. 1. (Color online) Phase diagram in the $\mathrm{Pe}_{R}-\phi$ plane in (a) 3D and (b) 2D. The color bar shows the active pressure scaled with the swim energy $k_{s} T_{s}=\zeta U_{0}^{2} \tau_{R} / 6$, and the blue and red curves are the binodal and spinodal, respectively. The critical point is shown with a red star. The open and filled symbols are simulation data with a homogeneous and phase-separated state, respectively.

The spinodal defines the regions of stability and is determined by setting $\partial \Pi^{\text {act }} / \partial \phi=0$. This is given by the red curve in Fig. 1 that passes through the extrema of each constantpressure isocontour ("isobar"). No notion of free energy is needed to obtain the spinodal-it is a purely mechanical quantity.

At the critical point $\partial \Pi^{\text {act }} / \partial \phi=\partial^{2} \Pi^{\text {act }} / \partial \phi^{2}=0$. In $3 D$ we find the critical volume fraction $\phi^{c} \approx 0.44$, active pressure $\Pi^{\text {act, } c} \phi^{c} /\left(n k_{s} T_{s}\right) \approx 0.21$, and Péclet number $\operatorname{Pe}_{R}^{c} \approx 0.028$, values consistent with our Brownian dynamics (BD) simulations. Like the spinodal, the critical point is identified using only mechanical arguments.

The blue curve in Fig. 1 delineates the "binodal" or coexistence regions, which we define as the equality of the chemical potential in the dilute and dense phases. Although the thermodynamic chemical potential is defined only for equilibrium systems, one can define a nonequilibrium chemical potential for active systems using standard macroscopic mechanical balances [7]: $n\left(\partial \mu^{\text {act }} / \partial n\right)=(1-\phi) \partial \Pi^{\text {act }} / \partial n$.

This definition agrees with the true thermodynamic chemical potential for molecular or colloidal solutes in solution [20] (see Appendix C). There are no approximations other than incompressibility of the solvent. Stress-induced diffusion, 


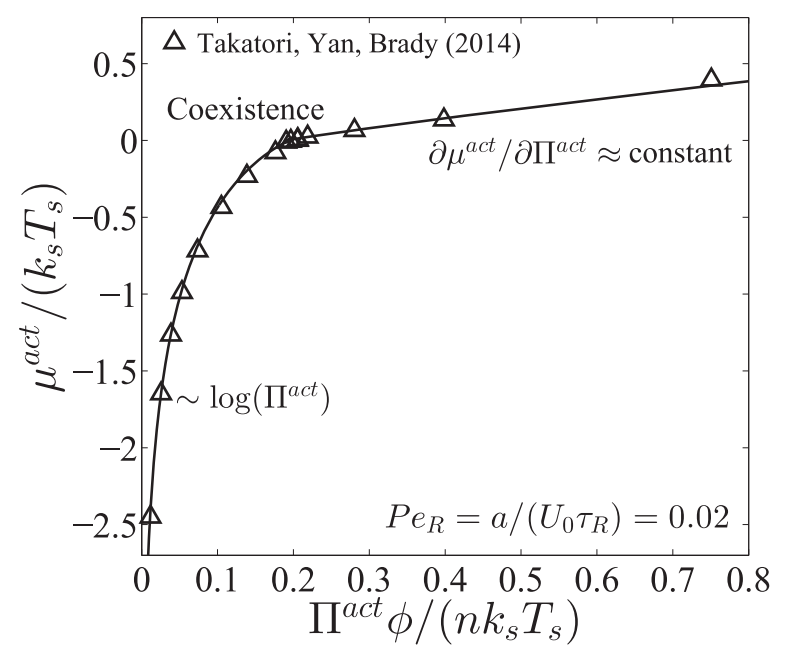

FIG. 2. Nonequilibrium chemical potential as a function of $\Pi^{\text {act }}$ for $\mathrm{Pe}_{R}=a /\left(U_{0} \tau_{R}\right)=\zeta U_{0} a /\left(6 k_{s} T_{s}\right)=0.02$, where $k_{s} T_{s}=\zeta U_{0}^{2} \tau_{R} / 6$ is the swimmers' energy scale. The symbols are BD simulations [7] and the curve is the model, Eq. (2).

which this relationship implies, has been used in the context of migration of non-Brownian particles in pressure-driven flow [21]. For $\tau_{R} \rightarrow 0$ active swimmers and passive Brownian particles not only behave similarly, but their dynamics are equivalent. If we placed active swimmers that behave identically to passive Brownian particles behind an osmotic barrier, we would not be able to distinguish one from the other. The form of the relationship between the chemical potential and pressure are equivalent for the two systems. We thus interpret $\mu^{\text {act }}$ as a natural definition and extension of the chemical potential for nonequilibrium systems, and use it to compute and define a binodal.

For small $\mathrm{Pe}_{R}$ we obtain

$$
\begin{aligned}
\mu^{\text {act }}\left(k_{s} T_{s}, \phi, \mathrm{Pe}_{R}\right)= & \mu^{\theta}\left(k_{s} T_{s}, \mathrm{Pe}_{R}\right)+k_{s} T_{s} \ln \phi \\
& +k_{s} T_{s} \ln \Gamma\left(\phi, \mathrm{Pe}_{R}\right),
\end{aligned}
$$

where $\mu^{\theta}\left(k_{s} T_{s}, \mathrm{Pe}_{R}\right)$ is the reference state whose form is not needed, and $\Gamma\left(\phi, \mathrm{Pe}_{R}\right)$ is a nonlinear but analytic expression [22]. The second term on the right-hand side represents the entropic, ideal-gas contribution to the chemical potential. The third term is the nonideal term that is the analog of enthalpic attraction between the active swimmers, and is represented by the quantity $\Gamma\left(\phi, \mathrm{Pe}_{R}\right)$ that resembles the fugacity coefficient in classical thermodynamics. Equation (2) is similar to that proposed by Cates and co-workers [2,23] who argued that $\mu(n)=\ln n+\ln v(n)$ where $v(n)$ is a density-dependent swimmer velocity. Our theory gives the nonideal contribution $\Gamma\left(\phi, \mathrm{Pe}_{R}\right)$ in the entire range of $\phi$ and $\mathrm{Pe}_{R}$.

The chemical potential from BD simulations and the model is shown in Fig. 2 for $\mathrm{Pe}_{R}=0.02$. It increases logarithmically at low $\Pi^{\text {act }}$ and the slope changes dramatically at the coexistence point $\left[\Pi^{\text {act }} \phi /\left(n k_{s} T_{s}\right) \approx 0.2\right]$. At this value of $\Pi^{\text {act }}$ and $\mathrm{Pe}_{R}$ the chemical potentials are equal in the dilute and dense phases. The data in the flat van der Waals region of the $\Pi^{\text {act }}-\phi$ phase diagram (see $\phi \approx 0.25-0.6$ in Fig. 2 of [7]) collapse onto the single coexistence point.
We can now define a binodal in Fig. 1 through the equality of the chemical potential in both phases. Our theory predicts that active systems prepared outside the binodal (blue curve) are stable in the homogeneous configuration and do not phase separate. The regions between the spinodal and binodal are metastable and a homogeneous system does not spontaneously phase separate via spinodal decomposition but can undergo a nucleation process. Nucleation times can be large and difficult to reach computationally, so artificial seeding may be required to induce phase separation [6].

As shown in Fig. 1(a) in 3D the transition from the homogeneous (open symbols) to phase-separated (filled symbols) systems in the simulations of Wysocki et al. [5] agree well with the spinodal of our model.

In two dimensions (2D), nucleation seeds form more easily compared to $3 \mathrm{D}$ because active swimmers self-assemble more easily in 2D-the colliding swimmers have fewer dimensions to "escape" the cluster (e.g., consider the extreme example of a one-dimensional system that readily clusters into a long string of swimmers). We surmise that nucleation processes are more likely to be observed in a $2 \mathrm{D}$ simulation prepared near the binodal curve. These observations are corroborated by Fig. 1(b) where we take the swim and interparticle pressures in $2 \mathrm{D}$ as $\Pi^{\text {swim }} /\left[n \zeta U_{0}^{2} \tau_{R} / 2\right]=1-\phi_{A}-0.2 \phi_{A}^{2}$ and $\Pi^{P} /\left(n \zeta U_{0}^{2} \tau_{R} / 2\right)=(4 / \pi) \phi_{A} \mathrm{Pe}_{R} g\left(2 ; \phi_{A}\right)$, respectively, where $\phi_{A}=n \pi a^{2}$ is the area fraction of active swimmers and $g\left(2 ; \phi_{A}\right)=\left(1-\phi_{A} / \phi_{0}\right)^{-\beta}$ with $\phi_{0}=0.9$ and $\beta=1$. The $2 \mathrm{D}$ simulation of Speck et al. [3] show that the transition from the homogeneous (open symbols) to phase-separated (filled symbols) states occur near the binodal (blue curve).

Our active pressure model agrees qualitatively and even quantitatively with the phase diagrams in Fig. 1, as well as with those of other studies $[2,4,6]$. It should be appreciated that there are no adjustable parameters in our theory.

\section{III. “THERMODYNAMIC” QUANTITIES}

The results presented thus far come from purely micromechanical arguments with no appeal to thermodynamics. We now turn our attention towards thermodynamic properties such as the free energy and temperature, which, although well defined for an equilibrium system, have been elusive for nonequilibrium systems.

Upon carefully imposing incompressibility of the solvent, one can relate the nonequilibrium Helmholtz free energy (FE) to the mechanical pressure as $\Pi^{\text {act }}\left(k_{s} T_{s}, \phi, \mathrm{Pe}_{R}\right)=\phi^{2}\left\{\partial / \partial \phi\left[\left(F^{\text {act }} / V\right) / \phi\right]\right\}$, where $V$ is the volume of the system [20]. There are again no approximations; it can be considered as the definition of the free energy for nonequilibrium active systems. Substituting the active pressure model for small $\mathrm{Pe}_{R}$ in $3 \mathrm{D}$, we obtain

$$
\begin{aligned}
F^{\text {act }}( & \left.\mathrm{Pe}_{R}<1\right) /\left(N k_{s} T_{s}\right) \\
= & \ln \phi-\phi(\phi+2) / 2-3 \mathrm{Pe}_{R} \phi_{0} \ln \left(1-\phi / \phi_{0}\right) \\
& +F^{\theta}\left(k_{s} T_{s}, \mathrm{Pe}_{R}\right),
\end{aligned}
$$

where $N$ is the number of active swimmers and $F^{\theta}\left(k_{s} T_{s}, \mathrm{Pe}_{R}\right)$ is the reference Helmholtz FE. The first term on the right can be interpreted as the ideal entropic contribution, and the rest represent the nonideal 


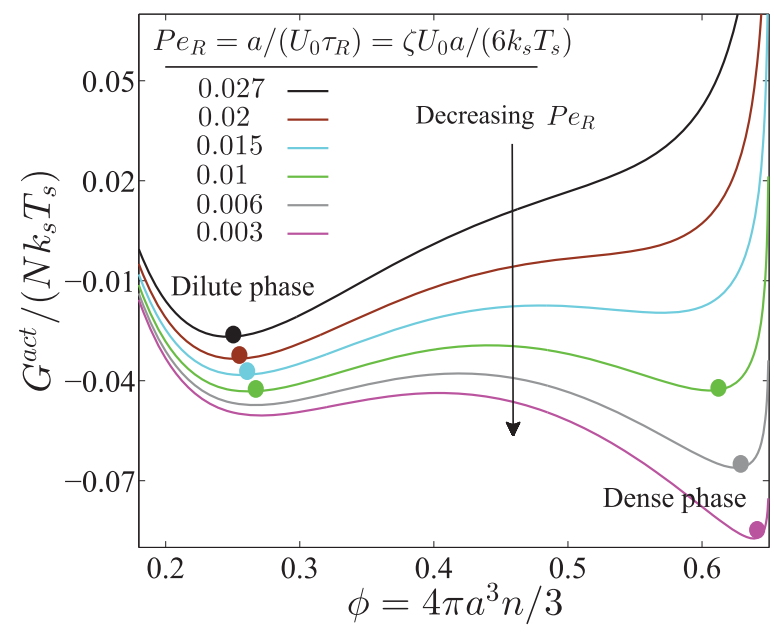

FIG. 3. (Color online) Gibbs FE as a function of $\phi$ for fixed values of $\mathrm{Pe}_{R}$ and $\Pi^{\mathrm{act}} \phi /\left(n k_{s} T_{s}\right)=0.18$, where $k_{s} T_{s}=\zeta U_{0}^{2} \tau_{R} / 6$. The black arrow points towards decreasing $\mathrm{Pe}_{R}$ at fixed $\Pi^{\text {act }}$. The filled color circles denote the stable states.

"enthalpic" attractions between the active swimmers. For large $\mathrm{Pe}_{R}$, the Helmholtz $\mathrm{FE}$ has no dependence on $\mathrm{Pe}_{R}$ : $F^{\mathrm{act}}\left(\mathrm{Pe}_{R}>1\right) /\left(N k_{s} T_{s}\right)=\ln \phi+4 \int_{0}^{\phi} g(2 ; s) d s+F^{\theta}\left(k_{s} T_{s}, \mathrm{Pe}_{R}\right)$, and has the same form as for Brownian hard-sphere systems. The Helmholtz FE, Eq. (3), has a form in agreement with Cates and co-workers $[2,23]$ who expressed the FE density as $f=n(\ln n-1)+\int_{0}^{n} \ln v(s) d s$.

Given a chemical potential we can further define the Gibbs FE as $\mu^{\text {act }}=\left(\partial G^{\text {act }} / \partial N\right)_{N_{f}, \Pi^{\text {act }}, T_{s}, \mathrm{Pe}_{R}}$, where $N_{f}$ is the number of solvent molecules [20]. Alternatively we can compute the Gibbs FE from the Helmholtz FE [20]: $G^{\text {act }} /\left(N k_{s} T_{s}\right)=F^{\text {act }} /\left(N k_{s} T_{s}\right)+\Pi^{\text {act }} /\left(n k_{s} T_{s}\right)$. Figure 3 shows the Gibbs FE as a function of $\phi$ for different values of $\mathrm{Pe}_{R}$ and fixed $\Pi^{\mathrm{act}} \phi /\left(n k_{s} T_{s}\right)=0.18$. As $\mathrm{Pe}_{R}$ decreases from a stable, dilute ideal-gas phase to $\mathrm{Pe}_{R}=0.015$ with a fixed $\Pi^{\text {act }} \phi /\left(n k_{s} T_{s}\right)=0.18, G^{\text {act }}$ has a local minimum at $\phi \approx 0.6$ corresponding to the metastable dense phase (i.e., "superheated liquid") and a global minimum at $\phi \approx 0.25$ corresponding to the stable dilute phase. At $\mathrm{Pe}_{R}=0.01$ the two minima of $G^{\text {act }}$ are equal corresponding to the coexistence of the dilute and dense phases.

By writing the ideal-gas swim pressure as $\Pi^{\text {swim }}=n \zeta U_{0}^{2} \tau_{R} / 6=n k_{s} T_{s}$, we can identify a swimmer's energy scale as $k_{s} T_{s}=\zeta U_{0}^{2} \tau_{R} / 6$. The reorientation Péclet number can be written as $\mathrm{Pe}_{R}=a /\left(U_{0} \tau_{R}\right)=\zeta U_{0} a /\left(6 k_{s} T_{s}\right)$, which is interpreted as a ratio of the interactive energy of the swimmer - the energy required to displace the swimmer, its size $a$-to the swim energy scale $k_{s} T_{s}$. Analogous to the Stokes-Einstein-Sutherland relation, one can interpret the swim diffusivity as $D^{\text {swim }}=k_{s} T_{s} / \zeta$, which also gives $\mathrm{Pe}_{R}=U_{0} a / D^{\mathrm{swim}} \sim \zeta U_{0} a /\left(k_{s} T_{s}\right)$.

From Fig. 1 phase separation occurs for small $\mathrm{Pe}_{R}=\zeta U_{0} a /\left(6 k_{s} T_{s}\right)$, or high $T_{s}$. This is opposite to what is typically observed in a classical thermodynamic system, where phase separation is driven by attractive enthalpic interactions and becomes possible at low temperatures. Phase separation with increasing temperature is uncommon but has been observed for systems driven by the lower critical solution temperature (LCST) transition [24,25] where phase transition is dominated by entropy. As $\mathrm{Pe}_{R}$ decreases ( $T_{s}$ increases) and the run length of the swimmer increases, the particle effectively becomes larger in size and thus has less space available for entropic mixing.

Many studies have discussed and proposed a possible interpretation of the temperature in a nonequilibrium active matter system [26-28]. Unlike a molecular fluid particle that is able to transmit its kinetic activity to another particle upon a collision, a self-propelled swimmer cannot impart its intrinsic activity to another swimmer. In this sense the swim temperature $T_{s}=\zeta U_{0}^{2} \tau_{R} /\left(6 k_{s}\right)$ is different from the temperature of an equilibrium thermal fluid because each swimmer has its own unique intrinsic kinetic temperature $T_{s}$ that does not get shared and equilibrated. Suppose we have a dilute suspension of completely inactive bath particles (i.e., not active swimmers nor Brownian particles). Into this we introduce a dilute concentration of active swimmers and monitor the motion of the passive bath particles. For small $\mathrm{Pe}_{R}$ the motion of the bath particles is not characterized by the swim diffusivity $D^{\text {swim }} \sim U_{0}^{2} \tau_{R}$ because the bath particles get a displacement of $\sim \mathcal{O}(a)$ upon colliding with a swimmer, not the run length $U_{0} \tau_{R}$. Thus, the diffusivity of the bath particles is $D^{\text {bath }} \sim \phi U_{0} a$, where $\phi$ is the volume fraction of the swimmers. The ratio of the bath to swimmer diffusivity is $D^{\text {bath }} / D^{\text {swim }} \sim \phi U_{0} a /\left(U_{0}^{2} \tau_{R}\right)=\phi \mathrm{Pe}_{R}$, suggesting that the reorientation Péclet number is the quantity that gets shared between the swimmers and not the swim energy $k_{s} T_{s}$.

The entropy of active matter can be defined as $S^{\text {act }}=-\left(\partial G^{\text {act }} / \partial T_{S}\right)_{\zeta U_{0} a, \Pi^{\text {act }}}=-\left(\partial F^{\text {act }} / \partial T_{S}\right)_{\zeta U_{0} a, \phi}$. Ignoring the reference states, for large $\mathrm{Pe}_{R}$ the entropy has the same form as that for a passive Brownian system: $S^{\text {act }}\left(\mathrm{Pe}_{R}>1\right) /\left(N k_{s}\right)=-\ln \phi-4 \int_{0}^{\phi} g(2 ; s) d s$. For small $\mathrm{Pe}_{R}$ the entropy comes solely from the swim pressure: $S^{\text {act }}\left(\mathrm{Pe}_{R}<1\right) /\left(N k_{s}\right)=-\ln \phi+\phi(\phi+2) / 2$. The entropy decreases with $\phi$ since the swimmers have less space available for entropic mixing.

The heat capacity can be obtained from $C_{V}=-T_{s}\left(\partial^{2} F^{\text {act }} / \partial T_{s}^{2}\right)_{\phi, \zeta U_{0} a}$. Aside from the reference state, substitution of the FE into this equation gives $C_{V}=0$ for all $\phi$ at both small and large $\mathrm{Pe}_{R}$. A possible explanation is that active matter has no true notion of the internal energy-since the swimmers cannot exchange their swim energy $k_{s} T_{s}$, there is no heat exchange between "hot" (high activity) and "cold" (low activity) active systems. There is no "first law" of thermodynamics for active matter systems [29].

In some experimental systems the swimmers may achieve self-propulsion by consuming and converting chemical fuel into mechanical motion. Swimmers may thus decrease their intrinsic swim velocity $\boldsymbol{U}_{0}$ when they are in a crowded region from the lack of fuel. This is a separate and independent effect as the reduction in the actual swimmer velocity $\boldsymbol{U}$ from collisions with other swimmers, which is already reflected in Eq. (4). Living microorganisms may possess an internal mechanism to detect changes in the local environment and alter their swim velocity or reorientation time. Hydrodynamics may also cause the drag factor to become density dependent. Our model remains applicable to swimmers with a local densitydependent intrinsic velocity $\boldsymbol{U}_{0}(\phi)$ and/or reorientation time 
$\tau_{R}(\phi)$. This effectively makes the swim temperature a function of the local volume fraction of active swimmers, $k_{s} T_{s}(\phi)-$ decreasing the chemical fuel concentration translates to decreasing the swim temperature of the system.

As shown in Appendix D, if we allow for a density (or position) -dependent intrinsic swim velocity $\boldsymbol{U}_{0}(\boldsymbol{x})$ and reorientation time $\tau_{R}(\boldsymbol{x})$, our definition of the nonequilibrium chemical potential becomes

$$
n \frac{\partial \mu^{\mathrm{act}}}{\partial n}=(1-\phi)\left[\frac{\partial \Pi^{\mathrm{act}}}{\partial n}-\Pi^{\mathrm{swim}}\left(\frac{\partial \ln \left(U_{0} \tau_{R}\right)}{\partial n}\right)\right] .
$$

Since $\Pi^{\text {act }}$ was determined for a homogeneous system, Eq. (1) still applies, but now $k_{s} T_{s}$ is also a function of $\phi$.

In active systems the relevant length scale is the swimmers' run length $U_{0} \tau_{R}$ and this must be small compared to the apparatus size in an experiment for the continuum approach to hold. In practice, experiments may have noncontinuum and nonlocal effects that may need to be considered when comparing experimental results with the thermodynamic model presented here.

In our model we neglected hydrodynamic interactions between the swimmers, which may contribute additional terms (such as the "hydrodynamic stresslet" [30]) to the active pressure, affect the reorientation time, and result in additional effects such as polar order of the swimmers. The ratio of the magnitudes of the hydrodynamic or polar stress to the swim stress is $\sim\left(n \zeta U_{0} a\right) /\left(n \zeta U_{0}^{2} \tau_{R}\right)=a /\left(U_{0} \tau_{R}\right) \equiv \mathrm{Pe}_{R}$; the hydrodynamic contribution becomes negligible when phase separation occurs for $\mathrm{Pe}_{R} \ll 1$ (see Fig. 1).

Much work remains to explore the implications of our "thermodynamics" of active matter and to see if it might apply to other far from equilibrium systems.

\section{ACKNOWLEDGMENTS}

S.C.T. is supported by a Gates Millennium Scholars fellowship and a National Science Foundation Graduate Research Fellowship (No. DGE-1144469). This work is also supported by NSF Grant No. CBET 1437570.

\section{APPENDIX A: MICROMECHANICAL EQUATIONS OF MOTION}

The active particle dynamics are governed by the $N$-particle Langevin equation

$$
\begin{gathered}
\mathbf{0}=-\zeta \boldsymbol{U}+\boldsymbol{F}^{\mathrm{swim}}+\boldsymbol{F}^{P}+\sqrt{2 \zeta^{2} D_{0}} \boldsymbol{\Lambda}_{T}, \\
\frac{d \boldsymbol{q}}{d t}=\sqrt{\frac{2}{\tau_{R}}} \boldsymbol{\Lambda}_{R} \times \boldsymbol{q},
\end{gathered}
$$

where $\boldsymbol{U}$ is the translational velocity, $\zeta$ is the hydrodynamic drag factor, $\boldsymbol{F}^{\text {swim }} \equiv \zeta \boldsymbol{U}_{0}=\zeta U_{0} \boldsymbol{q}$ is the self-propulsive swim force, $U_{0}$ is the swim speed, $\boldsymbol{q}$ is the unit vector specifying the swimmer's orientation, $\boldsymbol{F}^{P}$ is the interparticle force between the swimmers to enforce no overlap, $\boldsymbol{\Lambda}_{T}$ and $\boldsymbol{\Lambda}_{R}$ are unit random normal deviates, $\tau_{R}$ is the orientation time of the swimmer, and $D_{0}$ is the Stokes-Einstein-Sutherland translational diffusivity. The translational diffusivity and the reorientation dynamics are modeled with the usual white noise statistics, $\left\langle\Lambda_{i}(t)\right\rangle=0$ and $\left\langle\Lambda_{i}(t) \Lambda_{j}(0)\right\rangle=\delta(t) \delta_{i j}$. The left-hand side of Eq. (A1) is zero since inertia is negligible for colloidal suspensions. In this work we neglect the translational Brownian motion of active swimmers.

For $\tau_{R} \rightarrow 0$ active swimmers have small run lengths compared to their size and their dynamics are equivalent to that of passive Brownian particles. Indeed, an osmotic barrier cannot distinguish between a system of passive Brownian particles and active swimmers with small $\tau_{R}$. In this limit the form of the relationship between the pressure and other thermodynamic quantities (such as the chemical potential) are equivalent for the two systems.

\section{APPENDIX B: SWIM STRESS}

In [7] the swim stress was defined to be the first moment of the swim force

$$
\boldsymbol{\sigma}^{\text {swim }}=-n\left\langle\boldsymbol{x} \boldsymbol{F}^{\text {swim }}\right\rangle
$$

where $n$ is the number density of particles and the angle brackets denote an average over all particles and time. It is permissible for computing the stress to interpret the selfpropulsion of an active swimmer as arising from a swim force, $\boldsymbol{F}^{\text {swim }} \equiv \zeta \boldsymbol{U}_{0}$ [7]. This use of the swim force to compute the stress does not imply that the intrinsic swim mechanism generates a long-range $(1 / r)$ Stokes velocity field as does an external force [8].

The particle position at time $t$ is $\boldsymbol{x}(t)=\int \boldsymbol{U}\left(t^{\prime}\right) d t^{\prime}$, and from Eq. (A1) in the absence of interparticle forces (i.e., dilute suspension), we obtain $\boldsymbol{\sigma}^{\text {swim }}=-n\left\langle\boldsymbol{x} \boldsymbol{F}^{\text {swim }}\right\rangle=$ $-n \zeta \int\left\langle\boldsymbol{U}_{0}\left(t^{\prime}\right) \boldsymbol{U}_{0}(t)\right\rangle d t^{\prime}=-n \zeta \boldsymbol{D}^{\text {swim }}$, where the time integral of the intrinsic velocity autocorrelation is the swim diffusivity of the swimmer, $\boldsymbol{D}^{\text {swim }}$. Using the swim diffusivity $\boldsymbol{D}^{\text {swim }}=U_{0}^{2} \tau_{R} \boldsymbol{I} / 6$, we obtain the ideal-gas swim stress: $\boldsymbol{\sigma}^{\text {swim }}=-n \zeta U_{0}^{2} \tau_{R} \boldsymbol{I} / 6 \quad$ [7]. A dilute suspension of active swimmers therefore exerts a swim pressure, $\Pi^{\text {swim }}=-\operatorname{tr} \sigma^{\text {swim }} / 3=n \zeta U_{0}^{2} \tau_{R} / 6$, as given in the main text.

\section{APPENDIX C: MECHANICAL DERIVATION OF THE CHEMICAL POTENTIAL}

The number density of active swimmers satisfies the conservation equation

$$
\frac{\partial n}{\partial t}+\nabla \cdot \boldsymbol{j}=0
$$

where $\boldsymbol{j}=n \boldsymbol{u}_{p}=n\langle\boldsymbol{u}\rangle+\boldsymbol{j}^{\text {rel }}$ is the particle flux, $\boldsymbol{j}^{\text {rel }}=n\left(\boldsymbol{u}_{p}-\langle\boldsymbol{u}\rangle\right) \quad$ is the flux relative to the suspension average velocity $\langle\boldsymbol{u}\rangle$, which is defined as $\langle\boldsymbol{u}\rangle=\phi \boldsymbol{u}_{p}+(1-\phi) \boldsymbol{u}_{f}$, and $\boldsymbol{u}_{p}$ and $\boldsymbol{u}_{f}$ are the number averaged velocity of the swimmers and fluid at a continuum point, respectively. Due to incompressibility the suspension average velocity (particles plus the fluid) satisfies $\nabla \cdot\langle\boldsymbol{u}\rangle=0$.

To obtain an expression for $\boldsymbol{j}^{\text {rel }}$ we have no thermodynamic arguments to rely upon (such as the free energy) so we apply an averaged macroscopic mechanical momentum balance. Following the standard Irving-Kirkwood approach for averaging over a representative volume element as was done for non-Brownian suspensions [21], we obtain

$$
0=-n \zeta\left(\boldsymbol{u}_{p}-\langle\boldsymbol{u}\rangle\right)+\nabla \cdot \boldsymbol{\sigma}^{\mathrm{act}}
$$


where $\sigma^{\text {act }}=\sigma^{\text {swim }}+\sigma^{P}$ and the left-hand side is zero since inertia is negligible for colloidal systems. Using the relative flux $\boldsymbol{j}^{\text {rel }}=n\left(\boldsymbol{u}_{p}-\langle\boldsymbol{u}\rangle\right)$ we arrive at an explicit expression for the active particle flux in terms of gradients in the active stress:

$$
j^{\text {rel }}=\frac{1}{\zeta} \nabla \cdot \sigma^{\text {act }}
$$

No notion of a thermodynamic chemical potential or the free energy is needed to arrive at this expression. Substituting Eq. (C3) into the active particle conservation Eq. (C1), we obtain

$$
\frac{\partial n}{\partial t}+\langle\boldsymbol{u}\rangle \cdot \nabla n=-\nabla \cdot \frac{1}{\zeta} \nabla \cdot \boldsymbol{\sigma}^{\mathrm{act}},
$$

a convection-diffusion equation, where the diffusive nature is captured by gradients in the active stress. For a system that is macroscopically at rest, $\langle\boldsymbol{u}\rangle=\mathbf{0}$, and the active stress is isotropic, $\boldsymbol{\sigma}^{\text {act }}=-\Pi^{\text {act }} \boldsymbol{I}$, so Eq. (C4) becomes a diffusion equation

$$
\frac{\partial n}{\partial t}=\nabla \cdot \frac{1}{\zeta} \nabla \Pi^{\mathrm{act}} .
$$

This derivation is not restricted to active systems and applies equally well to equilibrium Brownian systems, where the Brownian osmotic pressure $\Pi^{B}=n k_{B} T$ gives

$$
\frac{\partial n}{\partial t}=\left(\frac{k_{B} T}{\zeta}\right) \nabla^{2} n
$$

a familiar diffusion equation with the Stokes-EinsteinSutherland translational diffusivity $D_{0}=k_{B} T / \zeta$. To continue the discussion of a passive Brownian system, which can be rigorously related to thermodynamic quantities, one can define a chemical potential precisely from a thermodynamic treatment [20] to give

$$
n \frac{\partial \mu^{B}}{\partial n}=(1-\phi) \frac{\partial \Pi^{B}}{\partial n} .
$$

In a thermodynamic system slightly out of equilibrium, the particle flux relative to the suspension average velocity is driven by the chemical potential gradient $\boldsymbol{j}^{\text {rel }}=-\{n /[(1-\phi) \zeta]\} \nabla \mu^{B}$. Comparing this flux expression with $\boldsymbol{j}^{\text {rel }}=-(1 / \zeta) \nabla \Pi^{B}$ [i.e., Eq. (C3) with $\sigma^{\text {act }}$ replaced by $\sigma^{B}=-\Pi^{B} I$ ] we arrive precisely at Eq. (C7).

Therefore the mechanical derivations of the stress, momentum balance, and flux are in full agreement with thermodynamics. In fact, one can analyze an equilibrium Brownian system purely from a mechanical perspective without appealing to thermodynamics [18]. Returning to active matter systems, we can rely upon the mechanical derivation to define a nonequilibrium chemical potential by analogy to the quantity whose gradient would drive a flux. Repeating the connection of the relative particle flux, $j^{\text {rel }}=-(1 / \zeta) \nabla \Pi^{\text {act }}$, to gradients in this newly defined chemical potential, $\quad \boldsymbol{j}^{\text {rel }}=-\{n /[(1-\phi) \zeta]\} \nabla \mu^{\text {act }}, \quad$ we arrive at $n\left(\partial \mu^{\text {act }} / \partial n\right)=(1-\phi) \partial \Pi^{\text {act }} / \partial n$, as used in the main text.

As mentioned in the main text, this relationship between the chemical potential and pressure are equivalent for a system of passive Brownian particles and active swimmers with small $\tau_{R}$. The dynamics of swimmers with $\tau_{R} \rightarrow 0$ is equivalent to that of passive Brownian particles, and one cannot distinguish between the two systems using confinement by an osmotic barrier. We thus interpret $\mu^{\text {act }}$ as a natural definition and extension of the chemical potential for nonequilibrium systems, and use it to compute and define a binodal.

\section{APPENDIX D: DENSITY-DEPENDENT SWIMMER ACTIVITY}

Suppose we have a density (or position) -dependent intrinsic swim velocity $\boldsymbol{U}_{0}(\boldsymbol{x})$ and reorientation time $\tau_{R}(\boldsymbol{x})$. These may vary spatially due to a variation in fuel concentration, for example. For a weak gradient we have

$$
\begin{aligned}
\boldsymbol{U}_{0}(\boldsymbol{x})= & U_{0}\left(\boldsymbol{x}_{0}\right) \boldsymbol{q}\left(\boldsymbol{x}_{0}\right)+\left(\nabla U_{0}\right)_{\boldsymbol{x}_{0}} \cdot\left(\boldsymbol{x}-\boldsymbol{x}_{0}\right) \boldsymbol{q}\left(\boldsymbol{x}_{0}\right) \\
& +U_{0}\left(\boldsymbol{x}_{0}\right)(\nabla \boldsymbol{q})_{\boldsymbol{x}_{0}} \cdot\left(\boldsymbol{x}-\boldsymbol{x}_{0}\right)+\cdots,
\end{aligned}
$$

where $\boldsymbol{q}$ is the unit orientation vector of the swimmer and the ellipsis contains all higher-order gradient terms. This gives rise to a drift velocity of the swimmers due to a nonzero average swim force $\left\langle\boldsymbol{F}^{\text {swim }}\right\rangle=\left\langle\zeta U_{0}(\boldsymbol{x}) \boldsymbol{q}\right\rangle=\zeta\left(\nabla U_{0}\right)_{x_{0}} \cdot\langle(\boldsymbol{x}-$ $\left.\left.\boldsymbol{x}_{0}\right) \boldsymbol{q}\left(\boldsymbol{x}_{0}\right)\right\rangle+\zeta U_{0}\left(\boldsymbol{x}_{0}\right)\left\langle(\nabla \boldsymbol{q})_{\boldsymbol{x}_{0}} \cdot\left(\boldsymbol{x}-\boldsymbol{x}_{0}\right)\right\rangle$, where we retain only the leading order in gradients. Using the swim stress $\boldsymbol{\sigma}^{\text {swim }}=-n\left\langle\boldsymbol{x} \boldsymbol{F}^{\text {swim }}\right\rangle=-n\left\langle\zeta U_{0}\left(\boldsymbol{x}-\boldsymbol{x}_{0}\right) \boldsymbol{q}\right\rangle$, we have

$$
\left\langle\boldsymbol{F}^{\mathrm{swim}}\right\rangle=-\frac{1}{n} \boldsymbol{\sigma}^{\mathrm{swim}} \cdot \nabla \ln \left(U_{0} \tau_{R}\right) .
$$

A nonzero average swim force impacts the macroscopic flux model by contributing an additional term to the expression for the relative particle flux [see Eq. (C3)]:

$$
\boldsymbol{j}^{\mathrm{rel}}=\frac{1}{\zeta}\left(\nabla \cdot \boldsymbol{\sigma}^{\mathrm{act}}+n\left\langle\boldsymbol{F}^{\mathrm{swim}}\right\rangle\right)
$$

where $\sigma^{\text {act }}=\sigma^{\text {swim }}+\sigma^{P}$ is the active particle stress. Substituting for the mean swim force we obtain a constitutive relation for active systems with a drift velocity:

$$
\boldsymbol{j}^{\mathrm{rel}}=\frac{1}{\zeta}\left[\nabla \cdot \boldsymbol{\sigma}^{\mathrm{act}}-\boldsymbol{\sigma}^{\mathrm{swim}} \cdot \nabla \ln \left(U_{0} \tau_{R}\right)\right] .
$$

For a dilute system of active swimmers, $\boldsymbol{\sigma}^{\text {act }} \approx \boldsymbol{\sigma}^{\text {swim }}=-n \zeta U_{0}^{2} \tau_{R} \boldsymbol{I} / 6$, and the relative flux becomes

$$
\boldsymbol{j}^{\mathrm{rel}}=-n D^{\mathrm{swim}}\left(\nabla \ln n+\nabla \ln U_{0}\right),
$$

where $D^{\text {swim }}=U_{0}^{2} \tau_{R} / 6$. This result agrees with the work of Cates and co-workers [14,23] who derived Eq. (D5) for a dilute system from consideration of the flux in a Smoluchowski analysis rather than from the swim stress perspective. And like Cates and co-workers [14,23] for a system with zero relative particle flux, we obtain the steady-state probability density $n(\boldsymbol{x}) \sim 1 / U_{0}(\boldsymbol{x})$. Notice that the $\nabla \ln \left(\tau_{R}\right)$ term cancels and Eq. (D5) does not change irrespective of $\tau_{R}(\boldsymbol{x})$ varying with position. For the general expression [Eq. (D4)] valid for all concentrations this may no longer be the case.

Repeating the connection of the relative particle flux, Eq. (D4), to gradients in the active chemical potential, $\boldsymbol{j}^{\text {rel }}=-\{n /[(1-\phi) \zeta]\} \nabla \mu^{\text {act }}$, we arrive at Eq. (4) of the main text. 
[1] J. Toner, Y. Tu, and S. Ramaswamy, Ann. Phys. (NY) 318, 170 (2005).

[2] J. Stenhammar, D. Marenduzzo, R. J. Allen, and M. E. Cates, Soft Matter 10, 1489 (2014).

[3] T. Speck, J. Bialké, A. M. Menzel, and H. Löwen, Phys. Rev. Lett. 112, 218304 (2014).

[4] Y. Fily, S. Henkes, and M. C. Marchetti, Soft Matter 10, 2132 (2014).

[5] A. Wysocki, R. G. Winkler, and G. Gompper, Europhys. Lett. 105, 48004 (2014).

[6] G. S. Redner, M. F. Hagan, and A. Baskaran, Phys. Rev. Lett. 110, 055701 (2013).

[7] S. C. Takatori, W. Yan, and J. F. Brady, Phys. Rev. Lett. 113, 028103 (2014).

[8] S. C. Takatori and J. F. Brady, Soft Matter 10, 9433 (2014).

[9] X. Yang, M. L. Manning, and M. C. Marchetti, Soft Matter 10, 6477 (2014).

[10] We use the conventional definition of the Péclet number as advection over diffusion, but others may use the inverse of this quantity.

[11] I. Theurkauff, C. Cottin-Bizonne, J. Palacci, C. Ybert, and L. Bocquet, Phys. Rev. Lett. 108, 268303 (2012).

[12] J. Palacci, S. Sacanna, A. P. Steinberg, D. J. Pine, and P. M. Chaikin, Science 339, 936 (2013).

[13] J. Bialké, H. Löwen, and T. Speck, Europhys. Lett. 103, 30008 (2013).

[14] J. Stenhammar, A. Tiribocchi, R. J. Allen, D. Marenduzzo, and M. E. Cates, Phys. Rev. Lett. 111, 145702 (2013).

[15] I. Buttinoni, J. Bialké, F. Kümmel, H. Löwen, C. Bechinger, and T. Speck, Phys. Rev. Lett. 110, 238301 (2013).
[16] D. McQuarrie, Statistical Mechanics (University Science Books, Mill Valley, California, 2000).

[17] W. Russel, D. Saville, and W. Schowalter, Colloidal Dispersions (Cambridge University Press, Cambridge, UK, 1992).

[18] J. F. Brady, J. Chem. Phys. 98, 3335 (1993).

[19] Pressure $p_{f}$ of the incompressible solvent is arbitrary.

[20] M. Doi, Soft Matter Physics (Oxford University Press, Oxford, UK, 2013).

[21] P. R. Nott and J. F. Brady, J. Fluid. Mech. 275, 157 (2006).

[22] The nonideal term for the nonequilibrium chemical potential in Eq. (2) is given by

$$
\begin{aligned}
\Gamma\left(\phi, \mathrm{Pe}_{R}\right)= & \left(1-\phi / \phi_{0}\right)^{-3 \phi_{0} \mathrm{Pe}_{R}} \exp \left[\phi^{3}-\phi^{2} / 2\right. \\
& \left.+3 \mathrm{Pe}_{R} \phi_{0}\left(1-\phi_{0}\right) /\left(1-\phi / \phi_{0}\right)-3 \phi\left(1-\phi_{0} \mathrm{Pe}_{R}\right)\right] .
\end{aligned}
$$

[23] J. Tailleur and M. E. Cates, Phys. Rev. Lett. 100, 218103 (2008).

[24] R. B. Griffiths and J. C. Wheeler, Phys. Rev. A 2, 1047 (1970).

[25] P. I. Freeman and J. S. Rowlinson, Polymer 1, 20 (1960).

[26] X.-L. Wu and A. Libchaber, Phys. Rev. Lett. 84, 3017 (2000).

[27] D. Loi, S. Mossa, and L. F. Cugliandolo, Soft Matter 7, 10193 (2011).

[28] M. E. Cates and J. Tailleur, Europhys. Lett. 101, 20010 (2013).

[29] The actual chemical energy consumed in propelling the swimmer is dissipated into the thermal bath of the solvent. The behavior of an active system depends on the activity $\zeta U_{0}$, not the actual energy consumed.

[30] D. Saintillan and M. J. Shelley, Phys. Fluids 20, 123304 (2008). 